\title{
Viabilidade econômica de diferentes sistemas de produção de carvão vegetal em escala industrial
}

\author{
Danilo Barros Donato ${ }^{1}$, Mateus Alves Magalhães ${ }^{1}$, Angelica de Cássia Oliveira Carneiro ${ }^{1}$, \\ Carlos Miguel Simões da Silva ${ }^{1}$, Wagner Davel Canal ${ }^{1}$, Márcio Lopes da Silva ${ }^{1}$ \\ ${ }^{1}$ Departamento de Engenharia Florestal, Universidade Federal de Viçosa (UFV), Viçosa, Brasil.
}

\begin{abstract}
RESUMO Este trabalho teve como objetivo realizar a análise de viabilidade econômica de quatro sistemas de produção de carvão vegetal em diferentes cenários de escala produtiva. Foram avaliados dois fornos retangulares, com volume interno de 380 e 700 $\mathrm{m}^{3}$, e dois fornos metálicos, com volume interno de 32 e $100 \mathrm{~m}^{3}$, considerando três cenários de produção - 2500; 5000 e 10000 metros cúbicos de carvão $(\mathrm{mdc})$ por mês. Para a estimativa do número necessário de fornos para cada cenário proposto, foram utilizados valores médios do rendimento gravimétrico e do ciclo de carbonização de cada forno avaliado, fornecidos pelas próprias empresas detentoras de cada tecnologia. Para a condução da análise financeira e econômica dos diferentes cenários estabelecidos, considerou-se um horizonte de planejamento de10 anos, e a taxa de juros de 7,5\% ao ano. A análise econômica foi realizada através da determinação dos seguintes indicadores: Valor Presente Líquido (VPL), Valor Anual Equivalente (VAE), Razão Benefício/Custo (B/C) e Lucratividade. Os indicadores econômicos de todos os cenários avaliados para os quatro tipos de fornos foram positivos e maiores que 1 , indicando que todos os projetos são economicamente viáveis. Os melhores indicadores foram apresentados pelo forno metálico com volume interno de $100 \mathrm{~m}^{3}$, no cenário de maior produção (10.000 mdc/mês), evidenciando a maior rentabilidade do projeto com relação aos demais.
\end{abstract}

Palavras-chave: cenários de produção; forno retangular; forno metálico; ciclo de carbonização.

\section{Economic viability of different charcoal production systems on an industrial scale}

\begin{abstract}
This study aimed to conduct a study of economic viability analysis of four charcoal production systems in different scenarios of production scale. It was evaluated two rectangular kilns, with an internal volume of 380 and $700 \mathrm{~m}^{3}$, and two metal kilns, with an internal volume of 32 and $100 \mathrm{~m}^{3}$ and considering three production scenarios - 2500; 5000 and 10,000 cubic meters of charcoal $(\mathrm{cmc})$ per month. To estimate the required number of kilns for each proposed scenario, it was used average values of gravimetric yield and carbonization cycle of each rated furnace, supplied by the companies themselves owners of each technology. For the conduct of financial and economic analysis of the different scenarios established, it was considered a planning horizon of 10 years and interest rate of $7.5 \%$ per year. The economic analysis was conduct by determining the following indicators: Net Present Value (NPV), Equivalent Annual Value (EAV), Benefit/Cost Ratio (B/C) and Profitability. The economic indicators of all scenarios evaluated for the four types of kilns were positive and greater than 1 , indicating that all projects are economically viable. The best indicators were presented by the metal kiln with an internal volume of $100 \mathrm{~m}^{3}$, the largest production scenario $(10,000 \mathrm{cmc} /$ month), showing the greater profitability of the project in relation to the others.
\end{abstract}

Keywords: production scenarios; rectangular kilns; metal kilns; carbonization cycle.

\section{Introdução}

A maior parte da produção de carvão vegetal no Brasil ainda é realizada por meio de fornos rudimentares de alvenaria e com baixo rendimento gravimétrico, em torno de $25 \%$ (PINHEIRO et al., 2006). A causa muitas vezes está na falta de automatização do processo de carbonização, sendo o controle realizado de maneira subjetiva pelos próprios carbonizadores. Além disso, esses fornos não possuem controle das emissões de gases poluentes, propiciando condições insalubres de trabalho dentro da unidade de produção e gerando 
sérios transtornos ambientais na região em que está instalada (ARRUDA et al., 2011).

Atualmente, o setor florestal investe em projetos de inovação tecnológica para encontrar sistemas alternativos de produção de carvão vegetal que possibilitem melhorias significativas nesse cenário (GUIMARÃES NETO, 2007).O desenvolvimento de fornos retangulares de alvenaria é, até o momento, o avanço tecnológico mais efetivo para o aumento em escala industrial da produtividade de carvão vegetal no Brasil. Esses fornos possuem alta capacidade de enfornamento de madeira, rendimentos gravimétricos superiores a 30\% (SANTOS e HATAKEYAMA, 2012) e possibilidade de mecanização das etapas de carregamento e descarregamento.

Além dos fornos de alvenaria, outros tipos estão sendo desenvolvidos, dentre os quais se destacam os fornos metálicos. Os fornos "bricarbras" e o DPC (DryingPyrolisisandCooling Secagem, pirólise e resfriamento) são alguns exemplos em escala industrial. O sistema de carbonização bricarbras utiliza fornos cilíndricos metálicos, que realizam a produção integrada de carvão vegetal em um sistema auxiliar de recuperação e aproveitamento dos gases da carbonização, com rendimento gravimétrico de 30 a 38\% (PINHEIRO et al., 2006). O sistema DPC, por sua vez, também permite o aproveitamento dos subprodutos da carbonização e o rendimento gravimétrico é de aproximadamente 40\% (CGEE, 2014).

Além da viabilidade técnica e ambiental de cada sistema de carbonização, a viabilidade econômica é o principal critério de escolha entre eles. O retorno financeiro com relação ao capital investido deve ser avaliado para a escolha correta do sistema a ser utilizado em cada cenário de produção desejado. Oliveira et al. (2013) e Souza et al. (2016), evidenciam que existem diferenças significativas entre os indicadores econômicos de cada tipo de forno e de cada escala produtiva, sendo necessário o estudo comparativo entre eles.
Diante o exposto, este trabalho teve como objetivo realizara análise de viabilidade econômica de quatro sistemas de produção de carvão vegetal - dois fornos retangulares de alvenaria e dois fornos metálicos - em diferentes cenários de escala produtiva.

\section{Material e Métodos}

O estudo baseou-se na simulação de projetos com fins de produção de carvão vegetal utilizando quatro diferentes sistemas de carbonização, com valores de produtividade, custos e receitas referentes ao ano de 2015. Para realização da análise econômica, foram estabelecidos três cenários de produção de carvão vegetal, sendo estes de 2500, 5000 e 10000 metros de carvão (mdc) por mês.

\section{Aspectos técnicos dos fornos}

Foram definidos os seguintes sistemas de carbonização considerando os diferentes cenários de produção: dois modelos de fornos retangulares de alvenaria - um com volume interno de $380 \mathrm{~m}^{3}$ e outro com $700 \mathrm{~m}^{3} \mathrm{e}$ dois modelos de fornos metálicos um com volume interno de $32 \mathrm{~m}^{3}$ e outro com 100 $\mathrm{m}^{3}$. O volume interno de cada forno corresponde a sua respectiva capacidade de enfornamento de madeira em metros estéreos (st), que considera os espaços vazios entre as toras.

Os valores referentes aos custos de construção e produção dos fornos retangulares foram fornecidos por uma empresa especializada na construção desses sistemas. Em relação a construção dos fornos retangulares, considerou-se que ambos possuem duas portas (uma de cada lado para facilitar as etapas de carregamento e descarregamento), sendo que para cada dois fornos terá um queimador dos gases da carbonização e que o sistema de admissão de ar para ambos os fornos 
será feito por "tatus". Já os valores dos custos dos fornos metálicos foram obtidos através de orçamentos das empresas fabricantes dos mesmos.

Para o cálculo do número de fornos a serem utilizados para cada cenário proposto, considerou as informações referentes ao rendimento gravimétrico médio de cada tipo de forno. Esses valores foram obtidos de empresas do setor florestal. Considerou-se um fator de conversão de estéreo de lenha (st) para $\mathrm{m}^{3}$ igual a 0,7 ; densidade média da madeira a ser carbonizada igual a $500 \mathrm{~kg} / \mathrm{m}^{3}$ e densidade média do carvão produzido igual a $200 \mathrm{~kg} / \mathrm{m}^{3}$. Esses valores utilizados estão baseados em resultados médios de análises laboratoriais de matérias genéticos destinados a produção de carvão vegetal.

Para a elaboração do fluxo de caixa de cada sistema produtivo, considerou-se R $\$ 37,83$ como o custo do estéreo (st) de madeira ao longo de todo o horizonte de planejamento. $\mathrm{O}$ preço médio foi obtido junto ao website do CIFlorestas para o ano de 2015 no estado de Minas Gerais.

\section{Análise financeira e econômica}

Para proceder à análise financeira e econômica dos projetos, considerou-se um horizonte de planejamento de10 anos e uma taxa de juros de 7,5\% ao ano, sendo que esta é a taxa de juros praticada acordo com Oliveira et al. (2013) e com o PRONAF florestal.

O Valor Presente Líquido (VPL) (Equação 1) é a diferença positiva entre receitas e custos, atualizados para determinada taxa de juros. Um projeto é viável, quando o VPL é maior que zero e quanto maior, mais atrativo é o projeto (REZENDE; OLIVEIRA, 2008).

$$
V P L=\sum_{j=0}^{n} R_{j}(1+i)^{-j}-\sum_{j=0}^{n} C_{j}(1+i)^{-j}
$$

Em que: $\mathrm{VPL}=$ valor presente líquido, em $\mathrm{R} \$ \mathrm{Rj}=$ receitas no período j, em $R$ \$ $C j=$ custos no período $j$, em $R$; i = taxa de desconto (\% ao ano); $\mathrm{j}=$ período de ocorrência de $\mathrm{Rj}$ e $\mathrm{Cj}$, ano; $\mathrm{n}=$ duração do projeto, em anos.

O Valor Anual Equivalente (VAE) pode ser entendido como uma parcela periódica e constante que paga o VPL do investimento em estudo ao longo do horizonte de planejamento (REZENDE; OLIVEIRA, 2008). Para o período de capitalização anual, utiliza-se a Equação 2.

$$
\mathrm{VAE}=\frac{\mathrm{VPL}(\mathrm{i})}{\left[1-(1+\mathrm{i})^{-\mathrm{n}}\right]}
$$

Em que: $\mathrm{VAE}=$ valor anual equivalente, em $\mathrm{R} \$ \mathrm{VPL}=$ valor presente líquido, em $\mathrm{R} \$ \mathrm{i}=$ taxa de desconto ( $\%$ ao ano); $\mathrm{n}=$ duração do projeto, em anos.

A Relação Beneficio/Custo (B/C) é obtida pela razão entre o valor presente dos benefícios e o valor presente dos custos para uma dada taxa de juros (Equação 3). Sendo a viabilidade de um projeto verificada quando o resultado é maior que 1 (REZENDE; OLIVEIRA, 2008).

$$
B / C=\frac{\sum_{j=0}^{n} R_{j}(1+i)^{-j}}{\sum_{j=0}^{n} C_{j}(1+i)^{-j}}
$$

Em que: $\mathrm{B} / \mathrm{C}=$ razão benefício/custo; $\mathrm{Rj}=$ receita final no ano j, em R\$; C j = custo final no ano j, em R\$; $i=$ taxa de desconto (\% ao ano); $\mathrm{j}=$ período de ocorrência de $\mathrm{Rj}$ e $\mathrm{Cj}$, ano; $\mathrm{n}=\mathrm{du}$ ração do projeto, em anos.

A lucratividade indica eficiência operacional de um projeto. É expressa como um valor percentual que aponta a proporção de ganhos de um negócio (Equação 4) (REZENDE; OLIVEIRA, 2008).

$$
\text { Lucratividade }=\frac{\text { Lucro Líquido }}{\text { Receita Total }} \times 100
$$

Em que: Lucro Líquido e Receita Total, em R\$.

De modo geral, os projetos que apresentam os indicadores econômicos positivos e maiores do que 1 são economicamente viáveis, sendo os que apresentarem os maiores valores 
são os que apresentam a melhor rentabilidade econômica, sendo por esses critérios descritos os mais indicados para implantação.

\section{Resultados e Discussão}

Na Tabela 1 são apresentados os dados técnicos para cada sistema de carbonização, de acordo com os cenários estabelecidos. Observa-se que os fornos retangulares de alvenaria apresentam maior capacidade de enfornamento, tanto em volume quanto em massa, em relação aos metálicos. No entanto, o ciclo de produção dos fornos metálicos é significativamente menor que o dos retangulares, o que compensa sua menor capacidade de enfornamento de madeira.

Segundo CGEE (2014), o rendimento gravimétrico deve ser levado em consideração para cálculos de área necessária de florestas plantadas e para sistemas de carbonização que promovem seu aumento, servindo como forma de contabilização de redução dos gases de efeito estufa.
O sistema de carbonização retangular 1, apesar de ter um maior ciclo de carbonização em relação aos demais, possui uma maior capacidade de enfornamento de madeira, logo a necessidade de um menor número de fornos. Por outro lado, o forno metálico2 possui uma menor capacidade de enfornamento, o que acarreta anecessidade de um maior número de fornos para que se atinja a produção estabelecida, o que gera também um aumento do número operações de carga e descarga de madeira.

Na Tabela 2 são apresentados os custos envolvidos nos diferentes sistemas de carbonização analisados. Observa-se que os custos de construção e manutenção dos fornos retangulares de alvenaria foram superiores aos dos fornos metálico. Já os maiores custos mensais com mão-de-obra foram apresentados pelo forno metálico 2 (32 st), em contrapartida os custos com equipamentos foram os menores. Os custos mensais com madeira foram maiores para o forno retangular $2(380$ st) e o forno metálico 2 (32 st).

Tabela 1. Informações técnicas dos diferentes sistemas de carbonização.

Table 1. Technical information of different carbonization systems.

\begin{tabular}{|c|c|c|c|c|c|c|}
\hline Forno & $\begin{array}{l}\text { Produção Mensal } \\
\text { de Carvão (mdc) }\end{array}$ & $\begin{array}{l}\text { Volume } \\
\text { Útil (st) }\end{array}$ & $\begin{array}{c}\text { Madeira/Forno } \\
(\mathrm{t})\end{array}$ & $\begin{array}{c}\text { Rendimento } \\
(\%)\end{array}$ & $\begin{array}{c}\text { Ciclo de Produção } \\
\text { (dias) }\end{array}$ & $\begin{array}{l}\text { Número de } \\
\text { fornos }\end{array}$ \\
\hline \multirow{3}{*}{ Retangular 1} & 2500 & 700 & 245,0 & 37 & \multirow{3}{*}{17} & 4 \\
\hline & 5000 & 700 & 245,0 & 37 & & 7 \\
\hline & 10000 & 700 & 245,0 & 37 & & 13 \\
\hline \multirow{3}{*}{ Retangular 2} & 2500 & 380 & 133,0 & 33 & \multirow{3}{*}{14} & 6 \\
\hline & 5000 & 380 & 133,0 & 33 & & 11 \\
\hline & 10000 & 380 & 133,0 & 33 & & 22 \\
\hline \multirow{3}{*}{ Metálico 1} & 2500 & 100 & 35,0 & 37 & \multirow{3}{*}{3} & 4 \\
\hline & 5000 & 100 & 35,0 & 37 & & 8 \\
\hline & 10000 & 100 & 35,0 & 37 & & 16 \\
\hline \multirow{3}{*}{ Metálico 2} & 2500 & 32 & 11,2 & 33 & \multirow{3}{*}{2,08} & 10 \\
\hline & 5000 & 32 & 11,2 & 33 & & 19 \\
\hline & 10000 & 32 & 11,2 & 33 & & 38 \\
\hline
\end{tabular}

Fonte: Elaborado pelos autores. 
Tabela 2. Custos envolvidos nos diferentes sistemas de carbonização.

Table 2. Costs involved in different carbonization systems.

\begin{tabular}{|c|c|c|c|c|c|}
\hline Forno & $\begin{array}{l}\text { Produção Mensal } \\
\text { (mdc) }\end{array}$ & $\begin{array}{c}\text { Construção + Manutenção do } \\
\text { forno }(\mathrm{R} \$)\end{array}$ & $\begin{array}{c}\text { Mão de Obra } \\
\text { (R\$/mês) }\end{array}$ & $\begin{array}{c}\text { Equipamento } \\
\text { (R\$) }\end{array}$ & $\begin{array}{l}\text { Madeira } \\
\text { (R\$/mês) }\end{array}$ \\
\hline \multirow{3}{*}{$\begin{array}{l}\text { Retangular } 1 \\
\quad \text { (700 st) }\end{array}$} & 2500 & $3.003 .000,0$ & $25.991,0$ & $200.000,0$ & $146.061,8$ \\
\hline & 5000 & $5.255 .250,0$ & $51.982,0$ & $400.000,0$ & $292.123,6$ \\
\hline & 10000 & $9.759 .750,0$ & $92.265,0$ & $600.000,0$ & $584.247,1$ \\
\hline \multirow{3}{*}{$\begin{array}{l}\text { Retangular } 2 \\
\quad \text { (380 st) }\end{array}$} & 2500 & $2.162 .160,0$ & $27.856,0$ & $200.000,0$ & $163.766,2$ \\
\hline & 5000 & $3.963 .960,0$ & $55.712,0$ & $400.000,0$ & $327.532,5$ \\
\hline & 10000 & $7.927 .920,0$ & $101.590,0$ & $600.000,0$ & $655.064,9$ \\
\hline \multirow{3}{*}{$\begin{array}{l}\text { Metálico } 1 \\
\text { (100 st) }\end{array}$} & 2500 & $1.605 .564,4$ & $20.396,0$ & $200.000,0$ & $146.061,8$ \\
\hline & 5000 & $3.211 .128,7$ & $38.927,0$ & $400.000,0$ & $292.123,6$ \\
\hline & 10000 & $6.422 .257,5$ & $66.155,0$ & $600.000,0$ & $584.247,1$ \\
\hline \multirow{3}{*}{$\begin{array}{l}\text { Metálico } 2 \\
\quad(32 \mathrm{st})\end{array}$} & 2500 & $872.812,5$ & $48.371,0$ & $100.000,0$ & $163.766,2$ \\
\hline & 5000 & $1.658 .343,8$ & $94.877,0$ & $200.000,0$ & $327.532,5$ \\
\hline & 10000 & $3.316 .687,5$ & $178.055,0$ & $300.000,0$ & $655.064,9$ \\
\hline
\end{tabular}

Os fornos retangulareslapresentam maior custo de construção por seu maior porte e por necessitarem de uma estrutura segura que suporte as carbonizações e também pelo fato de que as operações de carga e descarga serem mecanizadas.

O sistema metálico 1 por ser totalmente mecanizado e automatizado necessita de menos mão de obra em relação ao demais. Por outro lado, o metálico 2 apresenta alto custo com mão-de-obra por ter parte de suas operações manuais e pelo significativo número de fornos demandados para atingir a produções estabelecidas. Entretanto, esse sistema de carbonização demanda poucos equipamentos, reduzindo assim este tipo de custo.

Já em relação ao custo com madeira, esse é maior para os fornos retangular 2e metálico 2 , em função destes apresentarem menor rendimento gravimétrico em carvão vegetal, necessitando de uma maior quantidade de madeira para a mesma produção que os demais.

Sendo assim, com base nos aspectos técnicos e nos custos de produção envolvidos em cada sistema carbonização, obtiveram-se os indicadores econômicos, conforme apresentado na Tabela 3. O forno metálico 1apresentou os menores custos de produção e os melhores indicadores econômico de viabilidade. O cenário de maior escala produtiva (10.000 mdc/mês) apresentou a maior rentabilidade econômica para todos os sistemas de carbonização avaliados.

O forno metálico 2 apresentou o maior custo total em relação aos demais, já o sistema metálico 1 se mostrou menos oneroso, obtendo os melhores indicadores econômicos entre os sistemas avaliados. Observa-se que os indicadores aumentam com o aumento da escala de produção, reduzindo os custos. Os indicadores VPL e o VAE para todos os projetos avaliados foram positivos, indicando a viabilidade econômica dos projetos.

Os cálculos referentes à razão Benefício-Custo (B/C) foram superiores a 1 para todos os projetos analisados, sendo o sistema de carbonização metálico 1 o que obteve o maior valor para uma produção mensal de 10.000 mdc com a razão de 1,41 ; indicando que para esse projeto as receitas superam os custos em $41 \%$. 
Em relação à lucratividade, assim como nos demais índices econômicos o projeto do sistema de carbonização metálico 1 para uma produção de mensal de $10.000 \mathrm{mdc}$ obteve uma maior lucratividade, com valor de 32,36\%. Isso significa que para cada $\mathrm{R} \$ 100,00$ de carvão vegetal comercializado, $\mathrm{R} \$ 32,36$ é contabilizado como lucro.

Tabela 3. Indicadores econômicos e financeiros de diferentes sistemas de carbonização.

Table 3. Economic and financial indicators of different carbonization systems.

\begin{tabular}{|c|c|c|c|c|c|c|c|c|}
\hline Forno & $\begin{array}{l}\text { P.M. } \\
\text { (mdc) }\end{array}$ & $\begin{array}{c}\text { C.T. }{ }^{*} \mathrm{Mi}- \\
\text { lhões }(\mathrm{R} \$)\end{array}$ & $\begin{array}{c}\text { R.T. }{ }^{*} \text { Mi- } \\
\text { lhões (R\$) }\end{array}$ & $\begin{array}{c}\text { Custo } \\
(\mathrm{R} \$ / \mathbf{m d c})\end{array}$ & $\begin{array}{c}\text { VPL Mi- } \\
\text { lhões (R\$) }\end{array}$ & $\begin{array}{c}\text { VAE Mi- } \\
\text { lhões (R\$) }\end{array}$ & $\mathrm{B} / \mathrm{C}$ & $\begin{array}{c}\text { Lucro } \\
(\%)\end{array}$ \\
\hline \multirow{3}{*}{$\begin{array}{l}\text { Retangular } 1 \\
\quad(700 \mathrm{st})\end{array}$} & 2500 & 17,37 & 20,15 & 57,9 & 2,77 & 0,40 & 1,16 & 18,74 \\
\hline & 5000 & 34,00 & 40,29 & 56,7 & 6,29 & 0,92 & 1,19 & 20,02 \\
\hline & 10000 & 66,08 & 80,58 & 55,1 & 14,50 & 2,11 & 1,22 & 22,02 \\
\hline \multirow{3}{*}{$\begin{array}{c}\text { Retangular } 2 \\
\text { (380 st) }\end{array}$} & 2500 & 18,15 & 20,15 & 60,5 & 2,00 & 0,29 & 1,11 & 13,6 \\
\hline & 5000 & 35,93 & 40,29 & 59,9 & 4,36 & 0,64 & 1,12 & 14,22 \\
\hline & 10000 & 70,58 & 80,58 & 59,0 & 9,73 & 1,42 & 1,14 & 15,39 \\
\hline \multirow{3}{*}{$\begin{array}{l}\text { Metálico1 } \\
\text { (100 st) }\end{array}$} & 2500 & 15,52 & 20,15 & 51,7 & 4,63 & 0,67 & 1,30 & 25,79 \\
\hline & 5000 & 30,88 & 40,29 & 51,5 & 9,41 & 1,37 & 1,30 & 26,17 \\
\hline & 10000 & 60,60 & 80,58 & 50,5 & 19,99 & 2,91 & 1,33 & 27,54 \\
\hline \multirow{3}{*}{$\begin{array}{c}\text { Metálico } 2 \\
\text { (32 st) }\end{array}$} & 2500 & 18,45 & 20,15 & 61,5 & 1,70 & 0,25 & 1,09 & 9,95 \\
\hline & 5000 & 36,65 & 40,29 & 61,1 & 3,64 & 0,53 & 1,1 & 10,48 \\
\hline & 10000 & 72,24 & 80,58 & 60,2 & 8,34 & 1,22 & 1,12 & 11,76 \\
\hline
\end{tabular}

Sendo: P.M. - Produção média; C.T.* - Custos Totais corrigidos; R.T.* - Receita Total corrigida.

\section{Conclusões}

De acordo com as análises realizadas neste estudo e com os custos de produção e comercialização utilizados, pode-se concluir que:

Todos os sistemas de produção de carvão vegetal (fornos retangulares de alvenaria e fornos metálicos) em todos os cenários avaliados para escala produtiva foram economicamente viáveis de acordo com os indicadores de VPL, VAE, Benefício/Custo e Lucratividade.

Os melhores indicadores foram apresentados pelo forno metálico com volume interno de 100 st, no cenário de maior produção (10.000 mdc/mês), evidenciando a maior rentabilidade do projeto com relação aos demais.

\section{Agradecimentos}

Os autores agradecem à Fundação de Amparo à Pesquisa do Estado de Minas Gerais - FAPEMIG, à Secretaria de Estado, Ciência, Tecnologia e Ensino Superior - SECTES/ MG, ao Conselho Nacional de Pesquisa e Desenvolvimento $\mathrm{CNPq}$ pelo apoio financeiro.

\section{Referências}

ARRUDA, T. P. M.; PIMENTA, A. S.; VITAL, B. R.; LUCIA, R. M. D.; ACOSTA, F. C. Avaliação de duas rotinas de carbonização em fornos retangulares. Revista Árvore, Viçosa, MG, v. 35, n. 4, p. 949-955, 2011.

CGEE - Centro de Gestão e Estudos Estratégicos. Subsídios 2014 ao Plano-Siderurgia do MDIC: Modernização da produção de carvão vegetal. Brasília: Centro de Gestão e Estudos Estratégicos. 99p. 2014. 
GUIMARÃES NETO, R. M.; PIMENTA, A. S.; SILVA, M. L.; SOARES, N. S.; VITAL, B. R.; SILVA, J. C. Avaliação econômica e financeira de projetos de fornos dos tipos container industrial e retangular de 40 estéreos. Revista Árvore, Viçosa, MG, v. 31, n. 4, p. 709-715, 2007.

OLIVEIRA, A.C. et al. Viabilidade econômica da produção de carvão vegetal em dois sistemas produtivos. Floresta, v.44, n.1, p.143-152, 2013.

PINHEIRO, P. C. C.; REZENDE, M. E. A.;SAMPAIO, R. S. A produção de carvão vegetal: teoria e prática. Belo Horizonte, 2006.

REZENDE, J. L. P.; OLIVEIRA, A. D. Análise econômica e social de projetos florestais. 2. ed. Viçosa: UFV, 2008, 386 p.

SANTOS, S. F. O. M.; HATAKEYAMA, K. Processo sustentável de produção de carvão vegetal quanto aos aspectos: ambiental, econômico, social e cultural. Produção, v.22, n.2, p.309-321, 2012.

SOUZA, N.D. et al. Estudo de Caso de uma Planta de Carbonização: Avaliação de Características e Qualidade do Carvão Vegetal Visando Uso Siderúrgico. Floresta e Ambiente, v.23, n.2, p.270-277, 2016. 